\title{
Nitrogen incorporation into strained (In, Ga) (As, N) thin films grown on (100), (511), (411), (311), and (111) GaAs substrates studied by photoreflectance spectroscopy and high-resolution x-ray diffraction
}

\author{
J. Ibáñez ${ }^{a)}$ \\ Institut Jaume Almera, Consell Superior d'Investigacions Científiques (CSIC), Lluís Solé i Sabarís s/n, \\ 08028 Barcelona, Catalonia, Spain
}

R. Kudrawiec and J. Misiewicz

Institute of Physics, Wroclaw University of Technology, Wybrzeże Wyspiańskiego 27, 50-370 Wroclaw, Poland

M. Schmidbauer

Institute for Crystal Growth, Max-Born-Strasse 2, D-12489 Berlin, Germany

M. Henini

School of Physics and Astronomy, University of Nottingham, Nottingham NG7 2RD, United Kingdom

\author{
M. Hopkinson \\ Department of Electronic and Electrical Engineering, University of Sheffield, S3 3JD Sheffield, \\ United Kingdom
}

(Received 31 May 2006; accepted 30 August 2006; published online 13 November 2006)

\begin{abstract}
We have used photoreflectance (PR) and high-resolution x-ray diffraction (HRXRD) measurements to assess the composition of $\operatorname{In}_{x} \mathrm{Ga}_{1-x} \mathrm{As}_{1-y} \mathrm{~N}_{y}$ thin films $(x \sim 20 \%, y \sim 3 \%)$ grown by molecular beam epitaxy on GaAs substrates with different surface orientations. The aim of our work is to investigate the effect of substrate orientation on the incorporation of $\mathrm{N}$ and In into the films. Whereas in principle the composition of the $\operatorname{In}_{x} \mathrm{Ga}_{1-x} \mathrm{As}_{1-y} \mathrm{~N}_{y}$ films cannot be characterized by HRXRD alone because lattice spacings depend on both $x$ and $y$, we show that a combined analysis of the PR and HRXRD data allows us to determine the sample composition. Our data analysis suggests that the incorporation of $\mathrm{N}$ in $(\mathrm{In}, \mathrm{Ga})(\mathrm{As}, \mathrm{N})$ exhibits some dependence on substrate orientation, although not as strong as previously observed in $\mathrm{Ga}(\mathrm{As}, \mathrm{N})$. We determine shear deformation potentials for our samples that are sizably different than those obtained by linearly interpolating from the values of the pure binary compounds, which has already been observed in $\mathrm{Ga}(\mathrm{As}, \mathrm{N})$ and contradicts the currently accepted idea that only the conduction band of dilute nitrides is perturbed by N. ( $) 2006$ American Institute of Physics. [DOI: 10.1063/1.2374669]
\end{abstract}

\section{INTRODUCTION}

In recent years, much research effort has been devoted to dilute nitride $\mathrm{GaAs}_{1-y} \mathrm{~N}_{y}$ and $\mathrm{In}_{x} \mathrm{Ga}_{1-x} \mathrm{As}_{1-y} \mathrm{~N}_{y}$ alloys due to the unusual physical properties of these material systems. ${ }^{1}$ The large electronegativity of the $\mathrm{N}$ atoms combined with the stretching and compression of neighboring bonds in GaAs and $\mathrm{In}_{x} \mathrm{Ga}_{1-x}$ As strongly perturbs the band structure properties of the host crystals. An important manifestation of this perturbation is the huge band gap bowing with increasing $\mathrm{N}$ content and a strong redshift of the band gap. These electronic properties have significant potential to develop heterostructure devices such as $1.3 \mu \mathrm{m}$ lasers, solar cells, and heterojunction bipolar transistors. ${ }^{1}$

The growth of semiconductor layers and structures strongly depends on the surface atomic arrangement. Thus, it is expected that the incorporation of $\mathrm{N}$ into dilute nitrides depends on substrate orientation. Despite the interest of growing $\mathrm{GaAs}_{1-y} \mathrm{~N}_{y}$ and $\operatorname{In}_{x} \mathrm{Ga}_{1-x} \mathrm{As}_{1-y} \mathrm{~N}_{y}$ alloys on highindex surfaces, which could provide an additional means of tuning their optical emission, only a few works have ad-

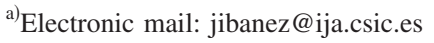

dressed the growth of these materials on non-(100) substrates. ${ }^{2-5}$ The interest of high-index substrates to grow dilute nitrides is even higher when mismatched growth is considered, since higher critical-layer thicknesses and longer emission wavelengths could be obtained. Also, the optoelectronic properties of non-(100) strained heterostructures are modified by the presence of strong built-in piezoelectric fields. ${ }^{6}$

In order to characterize and investigate the properties of dilute nitrides, one needs to determine with good accuracy the $\mathrm{N}$ content of the grown material. For this purpose, highresolution X-ray diffraction (HRXRD) measurements are widely used. In the case of $\mathrm{GaAs}_{1-y} \mathrm{~N}_{y}$, the HRXRD rocking curves allow determining $y$ if an assumption is made about the dependence of the lattice parameter and the elastic constants on $\mathrm{N}$ content. Usually, Vegard's law is assumed to analyze the HRXRD data, although there has been some controversy regarding its validity to determine the lattice parameter of dilute nitrides. ${ }^{7}$ Recent studies ${ }^{8,9}$ suggest that the lattice parameter of $\mathrm{GaAs}_{1-y} \mathrm{~N}_{y}$ only deviates from Vegard's law for $y \geq 3 \%$ and that such deviations are much lower than those reported previously. These studies suggest that the $\mathrm{N}$ 
composition measured by HRXRD may be underestimated for $y \geq 3 \%$ due to possible strain relaxation effects and the presence of interstitial $\mathrm{N}$ in $\mathrm{GaAs}_{1-y} \mathrm{~N}_{y}{ }^{8,9}$

In the case of $\operatorname{In}_{x} \mathrm{Ga}_{1-x} \mathrm{As}_{1-y} \mathrm{~N}_{y}$ epilayers, it is not possible in principle to determine simultaneously both the $\mathrm{N}$ and the In contents by means of HRXRD alone, since the lattice parameter of the quaternary alloy depends on both $x$ and $y$. As a consequence, one needs to determine independently the In or the $\mathrm{N}$ content in order to well characterize the material composition from the HRXRD data. For this end, secondaryion mass spectroscopy (SIMS) measurements are widely applied to determine both the $\mathrm{N}$ and In concentration of dilute nitride alloys. Other methods, such as Rutherford backscattering (RBS) channeling, cannot be applied to determine how $\mathrm{N}$ is incorporated into the lattice of dilute nitrides because $\mathrm{N}$ is a light ion, giving rise to a very low amount of backscattering which does not allow the analysis of the location and content of the $\mathrm{N}$ ions within the crystal lattice. In any case, both techniques are destructive and have a high error associated $(\sim 10 \%)$. In the case of SIMS, calibration standards are also required.

Alternatively, in order to determine the In and $\mathrm{N}$ contents of $\operatorname{In}_{x} \mathrm{Ga}_{1-x} \mathrm{As}_{1-y} \mathrm{~N}_{y}$ from the HRXRD data, one may assume that the In incorporation during growth is the same for (In, Ga)(As, N) than for (In, Ga)As, ${ }^{10,11}$ for which calibration of composition versus growth conditions should be available. However, there is no real knowledge of how the presence of $\mathrm{N}$ in the growth atmosphere affects the incorporation of In into $(\mathrm{In}, \mathrm{Ga})(\mathrm{As}, \mathrm{N})$. This becomes even more complex when high-index surfaces are considered. Thus, bearing in mind that the $\mathrm{N}$ content determined from the $\mathrm{x}$-ray rocking curves depends strongly on the In composition (i.e., small variations of $y$ give rise to large relative changes of the $x$ values obtained by HRXRD) it becomes essential to investigate the In incorporation in (In, Ga)(As, N) as a function of the growth direction. This type of study should confirm whether the HRXRD method alone is reliable enough to determine the sample composition when the In nominal composition is assumed.

In the present work we use photoreflectance (PR) spectroscopy, in combination with HRXRD measurements, to evaluate the $\mathrm{In}$ and $\mathrm{N}$ compositions of strained $\mathrm{In}_{x} \mathrm{Ga}_{1-x} \mathrm{As}_{1-y} \mathrm{~N}_{y} \quad(x \sim 20 \%, \quad y \sim 3 \%) \quad$ epitaxial thin films grown on (100), (511), (411), (311), and (111) GaAs substrates. PR measurements are one of the most widely used techniques to investigate dilute nitrides, providing a very good correlation between the measured band gaps and $\mathrm{N}$ composition through the band anticrossing (BAC) model. ${ }^{12,13}$

The PR spectra of the samples investigated here display two resonances corresponding to absorption between the heavy-hole $(\mathrm{HH})$ and light-hole $(\mathrm{LH})$ valence bands and the conduction band of (In, Ga)(As, N). We analyze the PR data by using the conventional perturbation treatment of the strain-induced valence-band shifts and splitting together with the BAC model. From the energy of LH and HH states, using the information about the lattice parameters provided by the HRXRD measurements, we evaluate the $\mathrm{N}$ and In content of the films and we obtain the shear deformation potentials of $\mathrm{In}_{x} \mathrm{Ga}_{1-x} \mathrm{As}_{1-y} \mathrm{~N}_{y}$ for $x \sim 20 \%, y \sim 3 \%$. Our results suggest that the incorporation of $\mathrm{N}$ in strained $(\mathrm{In}, \mathrm{Ga})(\mathrm{As}, \mathrm{N})$ depends on substrate orientation, although to a lesser extent than previously reported in $\mathrm{Ga}(\mathrm{As}, \mathrm{N}){ }^{2,3}$ Regarding the incorporation of In, we find that it only displays a weak dependence on the amount of $\mathrm{N}$ incorporated, which indicates that the HRXRD measurements alone may provide a fairly good approximation of the sample composition if the nominal In concentration is assumed. We find values for the shear deformation potentials, $b_{v}$ and $d_{v}$, sizably lower than those obtained by linearly interpolating between the values of the pure compounds. This result is in agreement with previous findings on $\mathrm{Ga}(\mathrm{As}, \mathrm{N}$ ) (Refs. 14 and 15) and, contrary to the accepted idea that $\mathrm{N}$ only perturbs the conduction band of dilute-nitride materials, it suggests that the valence band of dilute nitrides is also perturbed by $\mathrm{N}$.

\section{EXPERIMENT}

$\mathrm{In}_{x} \mathrm{Ga}_{1-x} \mathrm{As}_{1-y} \mathrm{~N}_{y}$ thin films $(x \sim 20 \%, y \sim 3 \%)$ were deposited by molecular beam epitaxy (MBE) on epiready GaAs substrates with the following orientations: (100), (511)A, (411)A, (311)A, and (111)A. To grow the samples, a $0.2-\mu \mathrm{m}$-thick undoped GaAs buffer layer was first deposited at $T=590{ }^{\circ} \mathrm{C}$ directly on the substrates. Then, the temperature was lowered to $T=400{ }^{\circ} \mathrm{C}$ and a $0.1-\mu \mathrm{m}$-thick undoped GaAs layer was deposited, followed by the $\operatorname{In}_{x} \mathrm{Ga}_{1-x} \mathrm{As}_{1-y} \mathrm{~N}_{y}$ layer. The nominal thickness of the $\operatorname{In}_{x} \mathrm{Ga}_{1-x} \mathrm{As}_{1-y} \mathrm{~N}_{y}$ films was $100 \mathrm{~nm}$.

The HRXRD measurements were performed with a double-crystal diffractometer, equipped with a parabolic (focusing) multilayer mirror and a four-bounce Ge 220 Bartels monochromator $(\mathrm{Cu} K \alpha 1$ line: $\lambda=1.5406 \AA)$. The angular resolution in the scattering plane was $\Delta \theta=11$ arc sec. Information about the thickness, crystal quality, the strain relaxation state, and the lattice parameter of the films was obtained from the HRXRD data.

Given that the calibration for MBE growth of (In, Ga)As was only available for the (100) orientation, we carried out $\mathrm{x}$-ray wavelength dispersion spectroscopy (WDS) measurements in order to confirm that the In fraction of all the $\mathrm{In}_{x} \mathrm{Ga}_{1-x} \mathrm{As}_{1-y} \mathrm{~N}_{y}$ layers was close to the nominal value $(x$ $\sim 20 \%$ ). For this purpose, a set of WDS measurements with different beam energies were acquired. The In fraction of the films was then inferred by analyzing the dependence on beam energy of the intensities of the different WDS peaks detected (peaks from In, $\mathrm{Ga}$, and As ions; emission from $\mathrm{N}$ ions was not detected). The WDS analysis was useful to corroborate that the In content of our (In, Ga)(As, N) films is around 20\%. However, the accuracy of the WDS results is fairly low (we estimate an error of $\sim 15 \%$ ) and, as a consequence, it is not possible to extract further information about the $\mathrm{N}$ content of the films by using both the WDS and the HRXRD data (see next section for details).

PR spectra were acquired by using a conventional experimental setup equipped with a tungsten halogen lamp $(150 \mathrm{~W})$ as a probe light source, a $0.55 \mathrm{~m}$ monochromator, and an InGaAs pin photodiode. For photomodulation, the $532 \mathrm{~nm}$ line of an yttrium aluminum garnet (YAG) laser with an output power of $15 \mathrm{~mW}$ was used. This pump beam was 

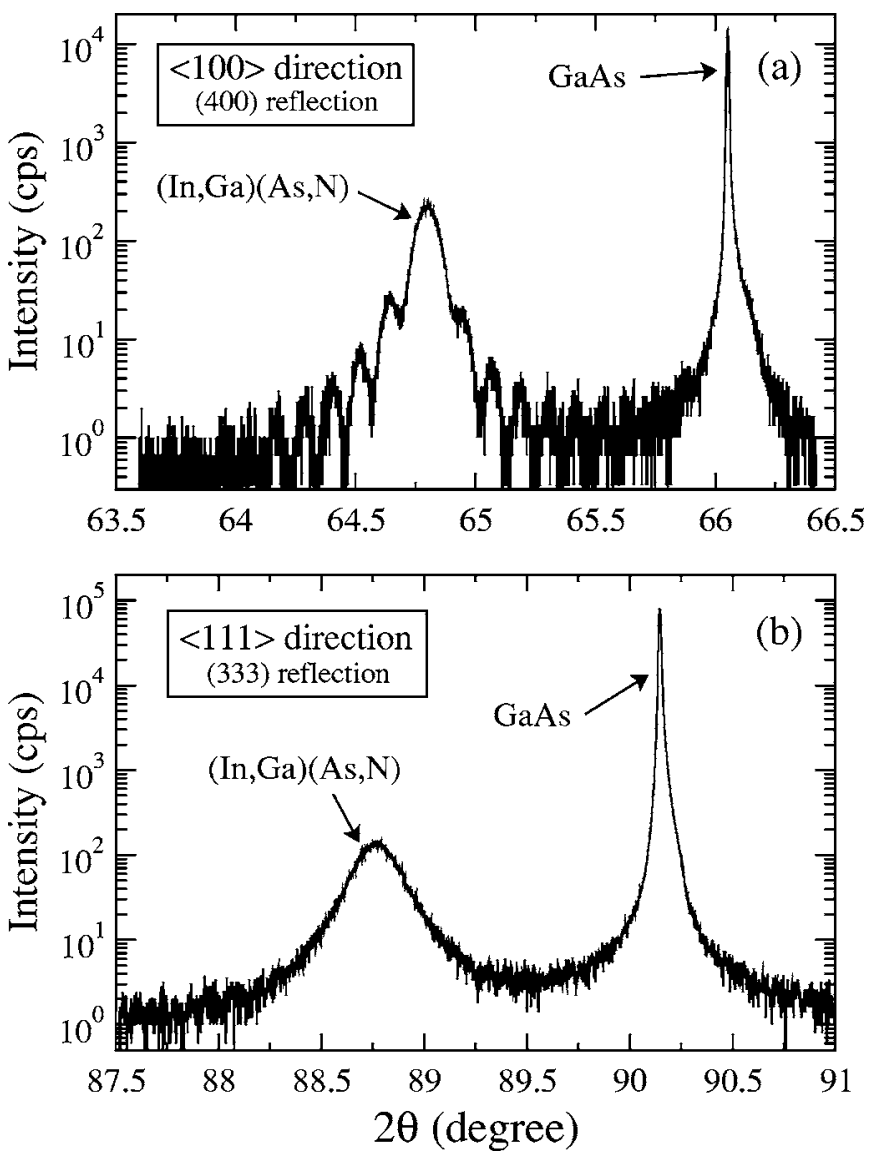

FIG. 1. X-ray rocking curves of two different (In, Ga)(As, N) thin films grown on (100)-GaAs [curve (a)] and on (111)-GaAs [curve (b)].

mechanically chopped at a frequency of $285 \mathrm{~Hz}$. The probe and pump beams were defocused to a diameter of $5 \mathrm{~mm}$. Phase sensitive detection of PR signal was made using a lock-in amplifier. Other relevant details of the PR setup are described elsewhere (Ref. 16).

\section{RESULTS}

\section{A. HRXRD measurements}

Figures 1(a) and 1(b) show selected x-ray rocking curves of the samples studied in this work. For the samples grown on (100)-, (111)-, (311)-, and (511)-GaAs the symmetrical (400), (333), (311), and (511) GaAs reflections, respectively, were chosen. In all the HRXRD rocking curves, besides the reflections corresponding to the GaAs substrate and GaAs buffer layers, reflections from the $(\mathrm{In}, \mathrm{Ga})(\mathrm{As}, \mathrm{N})$ films were detected. From the difference between the Bragg angles of the GaAs and the $(\mathrm{In}, \mathrm{Ga})(\mathrm{As}, \mathrm{N})$ reflections, the difference between the lattice spacings along the growth direction of the films and the GaAs substrates, $(\Delta d / d)_{\perp}$, was determined. The values thus obtained are displayed in the second column of Table I.

For the sample grown on (411)-GaAs, the asymmetrical (511) reflection of GaAs was considered. Two different geometries were used for this sample: (i) steep incidence and glancing exit and (ii) glancing incidence and steep exit. From these two measurements, both the horizontal and the vertical deformations were determined. The horizontal deformation of this film was found to be equal to zero, i.e., this sample is fully strained.

Regarding the strain state of the rest of samples, we first note that the rocking curves of the samples grown on (100)and (311)-GaAs substrates clearly display thickness fringes. This indicates that the quality of the $(\mathrm{In}, \mathrm{Ga})(\mathrm{As}, \mathrm{N}) / \mathrm{GaAs}$ interface for these two samples is good. The presence of thickness fringes suggests that these two (In, Ga)(As, N) layers are fully strained, since the density of dislocations in these samples is expected to be low.

For the samples grown on (511)- and (111)-GaAs, the HRXRD rocking curves do not display thickness fringes. To check the strain state of these two samples, a pair of rocking curves around asymmetrical reflections of GaAs were acquired (one measurement with glancing incidence and steep exit, and the other with steep incidence and glancing exit). These two measurements together provide information about the vertical and horizontal strain components of the films. For these samples, our measurements (not shown) indicated that, within experimental accuracy, the $(\mathrm{In}, \mathrm{Ga})(\mathrm{As}, \mathrm{N})$ layer is also fully strained.

Since all the $(\mathrm{In}, \mathrm{Ga})(\mathrm{As}, \mathrm{N})$ layers studied here are pseudomorphically grown, the values of $(\Delta d / d)_{\perp}$ obtained from the rocking curves (Table I) can be related to the relaxed lattice parameter of the films,

$$
a_{\mathrm{film}}=a_{\mathrm{GaAs}}\left[1+P\left(\frac{\Delta d}{d}\right)_{\perp}\right] \text {, }
$$

where $P$ is the elastic parameter of the films; ${ }^{17}$ regardless of the growth direction, the in-plane and out-of-plane biaxial strain components are given by $\epsilon_{\|}=\left(a_{\mathrm{film}}-a_{\mathrm{GaAs}}\right) / a_{\mathrm{GaAs}}$ and

TABLE I. List of samples studied in this work. The perpendicular lattice spacings $\left[(\Delta d / d)_{\perp, \text { HRXRD }}\right]$ determined by HRXRD, the energy and broadening of the HH and LH resonances detected by PR, and the In and N fractions as determined from the HRXRD and PR data are listed for all samples. Error bars for the experimental data are indicated in parentheses and refer to least significant digits [e.g., 0.01686 (14) is read as $0.01686 \pm 0.00014]$. On account of these experimental errors, the In fractions have been rounded to $\pm 0.5 \%$, while the $\mathrm{N}$ fractions have been rounded to $\pm 0.1 \%$.

\begin{tabular}{cccccccc}
\hline \hline Sample & $(\Delta d / d)_{\perp, \mathrm{HRXRD}}$ & $E_{\mathrm{HH}}(\mathrm{meV})$ & $\Gamma_{\mathrm{HH}}(\mathrm{meV})$ & $E_{\mathrm{LH}}(\mathrm{meV})$ & $\Gamma_{\mathrm{LH}}(\mathrm{meV})$ & {$[\mathrm{In}]$} & {$[\mathrm{N}]$} \\
\hline$(100)$ & $0.01686(14)$ & $857(1)$ & $29(1)$ & $904(1)$ & $37(1)$ & $22 \%$ & $3.7 \%$ \\
$(111) A$ & $0.01206(12)$ & $895(2)$ & $15(2)$ & $937(2)$ & $76(2)$ & $20.5 \%$ & $3.3 \%$ \\
$(311) A$ & $0.01543(15)$ & $914(1)$ & $20(1)$ & $962(1)$ & $38(1)$ & $20 \%$ & $2.8 \%$ \\
$(411) A$ & $0.01735(20)$ & $892(1)$ & $31(1)$ & $934(1)$ & $28(1)$ & $21.5 \%$ & $3.0 \%$ \\
$(511) A$ & $0.01736(17)$ & $950(3)$ & $41(3)$ & $1008(3)$ & $44(3)$ & $20 \%$ & $2.3 \%$ \\
\hline \hline
\end{tabular}


TABLE II. Values of the relaxed lattice parameter $(a)$, the elastic constants $\left(C_{i j}\right)$, and the deformation potentials $\left(a_{c}, a_{v}, b_{v}, d_{v}\right)$ of GaAs (Ref. 18), InAs (Ref. 18), InN (Ref. 19), and GaN (Ref. 19) used in this work to calculate the corresponding values of (In, Ga) (As, N).

\begin{tabular}{ccccc}
\hline \hline Parameters & GaAs & InAs & GaN & InN \\
\hline$a(\AA)$ & 5.65 & 6.06 & 4.50 & 4.98 \\
$C_{11}(\mathrm{GPa})$ & 1221 & 833 & 293 & 187 \\
$C_{12}(\mathrm{GPa})$ & 566 & 453 & 159 & 125 \\
$C_{44}(\mathrm{GPa})$ & 600 & 396 & 155 & 86 \\
$a_{c}(\mathrm{eV})$ & -7.17 & -5.08 & -6.71 & -2.65 \\
$a_{v}(\mathrm{eV})$ & -1.16 & -1.00 & -0.69 & -0.7 \\
$b_{v}(\mathrm{eV})$ & -2.0 & -1.8 & -2.0 & -1.2 \\
$d_{v}(\mathrm{eV})$ & -4.8 & -3.6 & -3.7 & -9.3 \\
\hline \hline
\end{tabular}

$\epsilon_{\perp}=\epsilon_{\|}(1-1 / P)$, respectively. For the sample grown on (100)-GaAs, $P$ is given by

$$
P_{100}=\frac{C_{11}}{C_{11}+2 C_{12}},
$$

whereas

$$
P_{111}=\frac{C_{11}+\frac{2}{3}\left(2 C_{44}-C_{11}+C_{12}\right)}{C_{11}+2 C_{12}}
$$

for the sample grown on (111)-GaAs. For the samples grown on $(N 11)$ substrates $(N=3,4$, or 5$)$, we obtain $P_{N 11}$ as a linear interpolation between the (100) and (111) orientations,

$$
P_{N 11}=P_{100}+\frac{\alpha}{54.74^{\circ}}\left(P_{111}-P_{100}\right)
$$

where $\alpha$ is the angle between the $\langle\mathrm{N} 11\rangle$ and $\langle 100\rangle$ directions and $\alpha=54.74^{\circ}$ is the angle between the $\langle 100\rangle$ and $\langle 111\rangle$ directions $\left[\alpha=15.79^{\circ}, 19.47^{\circ}\right.$, and $25.24^{\circ}$ for the (511), (411), and (311) layers, respectively]. This is a good approximation since the materials investigated in this study show a rather low elastic anisotropy. Throughout this work, we assume that the elastic constants $C_{i j}$ of $(\mathrm{In}, \mathrm{Ga})(\mathrm{As}, \mathrm{N})$ can be obtained by linearly interpolating from the values of the pure binary compounds. To obtain them, we use the values for GaAs and InAs provided in Ref. 18 and the values for zinc blende GaN and InN given in Ref. 19. For convenience, we display in Table II these values, together with the lattice parameters and deformation potentials of these compounds. ${ }^{18,19}$

If Vegard's law is assumed, the relaxed lattice parameter of the $\mathrm{In}_{x} \mathrm{Ga}_{1-x} \mathrm{As}_{1-y} \mathrm{~N}_{y}$ layers can be expressed as a function of $x, y$, and the lattice parameter of the (cubic) constituent components, ${ }^{20}$

$$
\begin{aligned}
a_{\mathrm{film}}= & x y a_{\mathrm{InN}}+x(1-y) a_{\mathrm{GaN}}-x y a_{\mathrm{InAs}}-x(1-y) a_{\mathrm{GaAs}} \\
& +y a_{\mathrm{InAs}}+(1-y) a_{\mathrm{GaAs}} .
\end{aligned}
$$

From this expression it is easy to see that the $\mathrm{In}$ and $\mathrm{N}$ concentration of the (In, Ga)(As, N) films cannot be determined independently from the values of $a_{\text {film }}$ obtained from the HRXRD data and Eq. (1). Yet, one could make an assumption about the In content of the films and take their nominal In concentration, $x \sim 20 \%$, to determine the $\mathrm{N}$ content. By following this procedure, we find that $y=2.85 \%$,

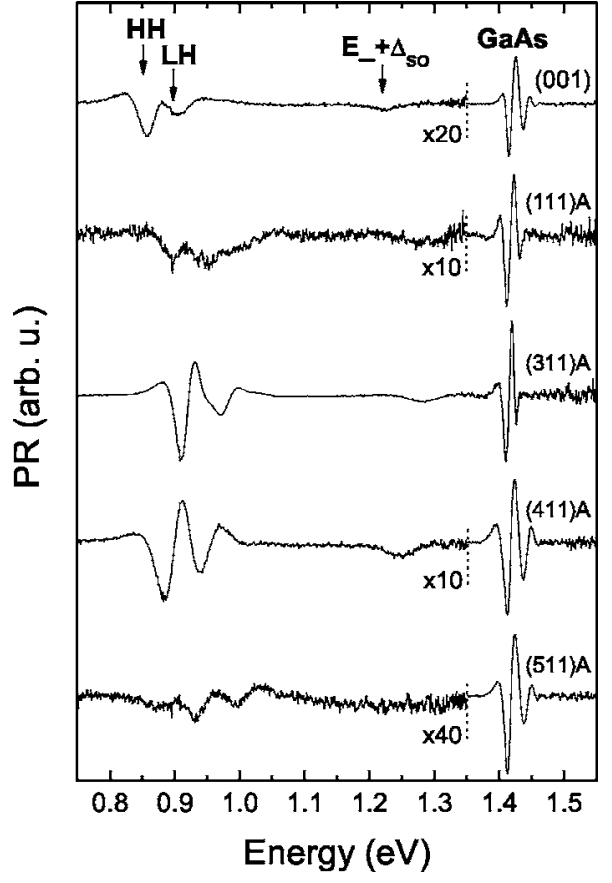

FIG. 2. PR spectra of the different $\operatorname{In}_{x} \mathrm{Ga}_{1-x} \mathrm{As}_{1-y} \mathrm{~N}_{y}$ thin films $(x \sim 20 \%, y$ $\sim 3 \%$ ) studied in this work.

$2.3 \%, 2.2 \%, 2.6 \%$, and $3.1 \%$ for our (100), (511), (411), (311), and (111) samples, respectively. However, if different values of $x$ are used to calculate $y$, we find that an absolute variation of $1 \%$ in the In fraction leads to a relative change of $y$ as high as $10 \%-15 \%$. Given this strong dependence of $y$ on $x$, and given that $x$ might depend on the growth direction, we conclude that the values of $y$ that we have obtained by taking $x=20 \%$ are not reliable. In other words, it is necessary to check with an alternative method whether $x \sim 20 \%$ in our samples to confirm that the HRXRD data alone provides an accurate determination of the composition of the (In, Ga)(As, N) layers.

\section{B. PR measurements}

Figure 2 shows room-temperature PR spectra of the samples investigated in this work. Besides the resonances corresponding to the GaAs buffer layers and/or substrate, all spectra display two clear resonances in the $0.8-1.0 \mathrm{eV}$ region. These resonances are originated by absorption between the $\mathrm{HH}$ and $\mathrm{LH}$ valence bands and the conduction band of $(\mathrm{In}, \mathrm{Ga})(\mathrm{As}, \mathrm{N})$. The energy separation between these two features is a consequence of the splitting of the valence band due to the compressive elastic strain of the layers. In the $1.2-1.3 \mathrm{eV}$ region of all spectra, a weak and broad band, which can be assigned to absorption between the split-off spin-orbit band and the conduction band, is also detected.

To extract the energy of the optical transitions, the PR resonances were analyzed using the low-field electromodulation Lorentzian line-shape functional form, ${ }^{21,22}$

$$
\frac{\Delta R}{R}(E)=\operatorname{Re}\left[\sum_{j=1}^{n} C_{j} \exp ^{\imath \theta_{j}}\left(E-E_{j}+\imath \Gamma_{j}\right)^{-m_{j}}\right],
$$

where $n$ is the number of optical transitions and spectral functions used in the fitting procedure; $C_{j}$ and $\theta_{j}$ are the 


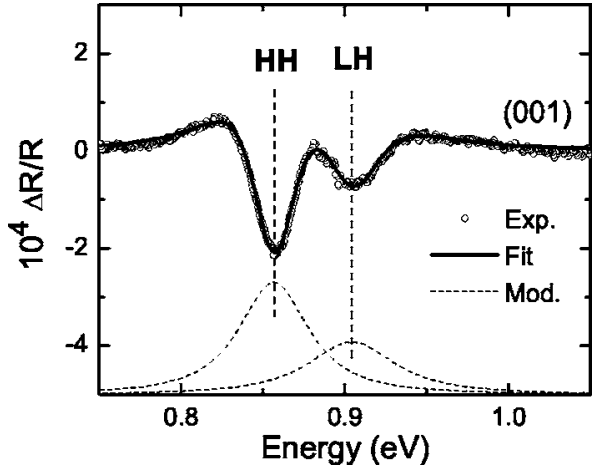

FIG. 3. Fitting curve (solid line), calculated with a low-field electromodulation Lorentzian line shape, corresponding to the PR spectrum of the (In, Ga)(As, N) sample grown on (100)-GaAs (dots). The modulus of the individual resonances, $\mathrm{HH}$ and $\mathrm{LH}$, are plotted with dashed lines.

amplitude and phase of the line shape, and $E_{j}$ and $\Gamma_{j}$ are the energy and the broadening parameter of the transitions, respectively. The parameter $m_{j}$ refers to the type of optical transition. For a three-dimensional (3D) one-electron transition, $m_{j}=5 / 2$.

An example of the fitting analysis, performed for the sample grown on a (100)-GaAs substrate, is shown in Fig. 3. The fitting curve that we obtain for the $\mathrm{LH}$ and $\mathrm{HH}$ features is plotted with a thick line. In addition, we plot by dashed lines in the figure the modulus of the individual resonances, which are obtained with the following expression:

$$
\left|\Delta \rho_{j}(E)\right|=\frac{\left|C_{j}\right|}{\left[\left(E-E_{j}\right)^{2}+\Gamma_{j}^{2}\right]^{m / 2}}
$$

with $C_{j}, E_{j}$, and $\Gamma_{j}$ parameters taken from the fit. The energy and the broadening parameter of the $\mathrm{HH}$ and $\mathrm{LH}$ resonances as obtained with this fitting procedure are displayed in Table I. In the next section, we use the energy of these two resonances in combination with the HRXRD data to determine the In and $\mathrm{N}$ content of our samples.

\section{DETERMINATION OF THE N AND IN CONTENT}

\section{A. (In, Ga)(As, N) grown on (100)-GaAs}

As discussed in the previous section, two resonances, $\mathrm{LH}$ and $\mathrm{HH}$, are detected by $\mathrm{PR}$ in the strained $\mathrm{In}_{x} \mathrm{Ga}_{1-x} \mathrm{As}_{1-y} \mathrm{~N}_{y}$ epilayers. The strain state (i.e., the lattice parameter) and the energy of these two resonances, $E_{\mathrm{LH}}$ and $E_{\mathrm{HH}}$, are determined solely by $x$ and $y$. As a consequence, $E_{\mathrm{LH}}$ and $E_{\mathrm{HH}}$ can be calculated as a function of $x$ and $y$ by using the conventional perturbation treatment of the straininduced valence-band shifts and splitting, provided that the composition dependence of the band gap of the unstrained material is known. Then, the energy of LH and HH determined from the PR measurements, in combination with the lattice parameters obtained by HRXRD, can be used to assess both $x$ and $y$ in the (In, Ga)(As, N) films.

The unstrained band gap of $\operatorname{In}_{x} \mathrm{Ga}_{1-x} \mathrm{As}_{1-y} \mathrm{~N}_{y}$ can be calculated as a function of $y$ by using the BAC model, which has been shown to reproduce the dependence on $\mathrm{N}$ content of the band-gap energy of dilute nitrides. ${ }^{12,13}$ Within this formalism, the conduction band of dilute nitrides is described in terms of the interaction of the localized level $\left(E_{N}\right)$ introduced by $\mathrm{N}$ ions with the condution band $\left(E_{M}\right)$ of the matrix material. The admixing of the localized states with the conduction band states gives rise to two conduction subbands, $E_{-}$and $E_{+}$, the energy of which is given by ${ }^{13}$

$$
E_{ \pm}(x, y)=\frac{1}{2}\left[E_{M}+E_{N} \pm \sqrt{\left(E_{M}-E_{N}\right)^{2}+4 y C_{M N}^{2}}\right],
$$

where $C_{M N}(x)=2.7(1-x)+2 x \mathrm{eV}$ and $E_{N}(x)=1.65-0.4 x(1$ $-x) \mathrm{eV}$ in the case of $\operatorname{In}_{x} \mathrm{Ga}_{1-x} \mathrm{As}_{1-y} \mathrm{~N}_{y}{ }^{13}$ To calculate the band-gap energy of unstrained (In, Ga)(As, N) [i.e., $\left.E_{-}(x, y)\right]$, we take the band parameters for the (In, Ga)As ternary alloy given in Ref. 18, where a bowing parameter $C=0.477 \mathrm{eV}$ is proposed for the dependence on In composition of the fundamental gap $\left(E_{M}\right)$ of this material. We take room-temperature values for the band-gap energies of GaAs and $\operatorname{InAs}, E_{g}(\mathrm{GaAs})=1.428 \mathrm{eV}$ and $E_{g}(\mathrm{InAs})=0.355 \mathrm{eV}{ }^{18}$

In order to calculate the energy of the $\mathrm{LH}$ and $\mathrm{HH}$ resonances observed by PR in our strained (In, Ga)(As, N) films, we need to compute the effect of strain on the conductionand valence-band states of the material. For the sample grown on (100)-GaAs, the energy of the HH and LH resonances is given, up to first order in the strain, by ${ }^{23,24}$

$$
\begin{aligned}
& E_{\mathrm{HH}}=E_{-}(x, y)-2\left(a_{c}+a_{v}\right)\left(\frac{C_{11}-C_{12}}{C_{11}}\right) \epsilon_{\|}+\frac{1}{2} \delta E_{100}, \\
& E_{\mathrm{LH}}=E_{-}(x, y)-2\left(a_{c}+a_{v}\right)\left(\frac{C_{11}-C_{12}}{C_{11}}\right) \epsilon_{\|}-\frac{1}{2} \delta E_{100},
\end{aligned}
$$

where

$$
\delta E_{100}=2 b_{v}\left(\frac{C_{11}+2 C_{12}}{C_{11}}\right) \epsilon_{\|}
$$

is the HH-LH splitting. $a_{c}$ and $a_{v}$ are the conduction-band and valence-band hydrostatic deformation potentials, and $b_{v}$ is the uniaxial shear deformation potential for the strain components with tetragonal symmetry. ${ }^{22}$ As discussed above, $E_{-}(x, y)$ is the band gap of unstrained $\operatorname{In}_{x} \mathrm{Ga}_{1-x} \mathrm{As}_{1-y} \mathrm{~N}_{y}$, computed with the BAC model [Eq. (8)]. For (100) samples, $\epsilon_{\|}$ $=-\epsilon_{x x}=-\epsilon_{y y}$.

In Fig. 4, we show the calculated energy of the $\mathrm{HH}$ and LH resonances of $\operatorname{In}_{x} \mathrm{Ga}_{1-x} \mathrm{As}_{1-y} \mathrm{~N}_{y}$ coherently grown on (100)-GaAs, obtained with Eqs. (9) and (10), for three different values of the In content $(x=21 \%, 22 \%$, and 23\%) as a function of $y(3 \%<y<4 \%)$. All the curves were obtained by first determining from the HH-LH energy splitting measured by PR (47 meV) and Eq. (11) the shear deformation potential, $b_{v}$, as a function of $x$ and $y$. The strain tensor components were obtained by taking values for the elastic constants of $(\mathrm{In}, \mathrm{Ga})(\mathrm{As}, \mathrm{N})$ that vary linearly from those of pure InAs, GaAs, cubic GaN, and cubic InN (Table II). As expected, the calculated energies of both $\mathrm{LH}$ and $\mathrm{HH}$ resonances decrease with increasing $\mathrm{N}$ or In content.

As can be observed in the figure, the calculated values of $E_{\mathrm{HH}}$ and $E_{\mathrm{LH}}$ coincide with the experimental values $\left(E_{\mathrm{LH}}\right.$ $=904 \mathrm{meV}$ and $E_{\mathrm{LH}}=857 \mathrm{meV}$ ) for different combinations of $x$ and $y$ and, as a consequence, it is not possible to determine $x$ and $y$ by using only the PR data. However, we find 


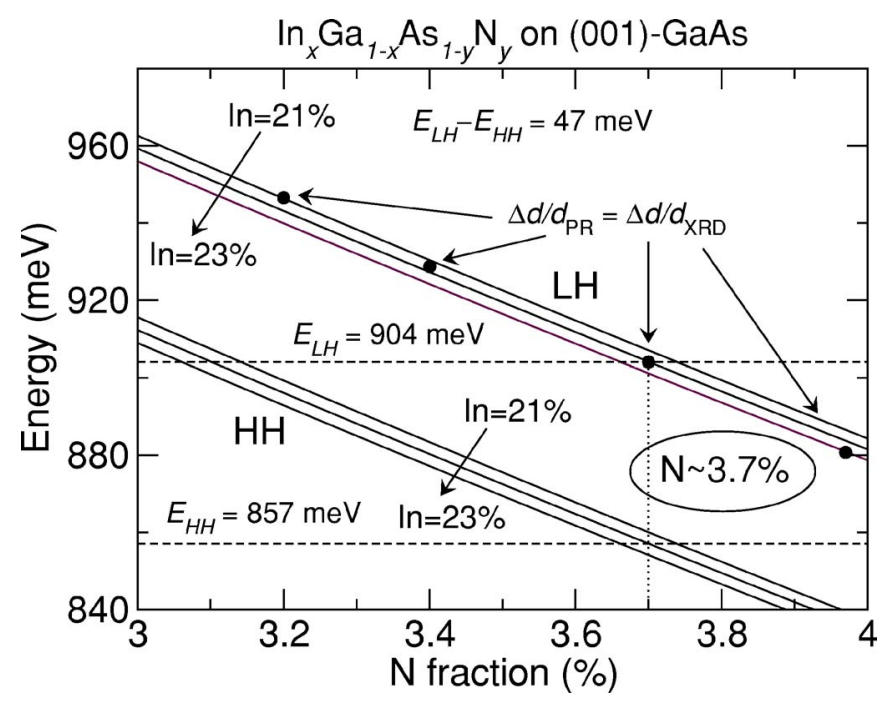

FIG. 4. Calculated energy values for the $\mathrm{LH}$ and $\mathrm{HH}$ resonances of $\mathrm{In}_{x} \mathrm{Ga}_{1-x} \mathrm{As}_{1-y} \mathrm{~N}_{y}$ coherently grown on (100)-GaAs as a function of $y$ for $x$ $=21 \%, 22 \%$, and $23 \%$. The solid circles indicate combinations of $y$ and $E_{\mathrm{LH}}(x, y)$ values that are compatible with the lattice spacing obtained by HRXRD for the (100) sample.

that only when $x \sim 22 \%$ and $y \sim 3.7 \%$ the resulting lattice parameter of $\operatorname{In}_{x} \mathrm{Ga}_{1-x} \mathrm{As}_{1-y} \mathrm{~N}_{y}$ grown on GaAs is the same as that determined with the HRXRD measurements. To illustrate this, we plot with solid circles in Fig. 4 several combinations of $\mathrm{N}$ content and $E_{\mathrm{LH}}(x, y)$ values that yield a lattice spacing equal to that determined by $\operatorname{HRXRD}\left[(\Delta d / d)_{\perp}\right.$ $=0.01686]$. As can be seen in the figure, only when $x$ $\sim 22 \%$ and $y \sim 3.7 \%$ the calculated $\mathrm{LH}$ and $\mathrm{HH}$ energies are in agreement with the experimental values obtained by PR and with the HRXRD data for the (100) sample. This allows us to infer the $\mathrm{N}$ and In concentration of this sample.

For this composition, taking into account the HH-LH splitting measured by PR, we find that the shear deformation potential is $b_{v}=-1.43$. This value is substantially lower than that one would obtain by linearly interpolating from the values of the pure binary compounds $\left(b_{v}=-1.95\right.$, see Table II). This result is in agreement with previous works, which reported marked differences between measurements of $b_{v}$ and the linearly interpolated values in $\mathrm{Ga}(\mathrm{As}, \mathrm{N}){ }^{14,15}$ Such differences suggest that the valence band of dilute nitrides is perturbed by $\mathrm{N}$ incorporation. As discussed in Ref. 14, this effect might be originated by lattice relaxation around the nitrogen impurities, which would give rise to additional shear strains that modify the valence-band states. More investigations are required to fully understand this surprising property of dilute nitrides.

\section{B. $($ In, Ga) $($ As, N) grown on (111) A-GaAs}

For strained epilayers grown on (111) substrates, the analysis is almost identical to that for the (100) sample. For this case, $\delta E_{100}$ in Eqs. (9) and (10) has to be replaced by ${ }^{23}$

$$
\delta E_{111}=d_{v} \sqrt{3}\left(\frac{C_{11}+2 C_{12}}{2 C_{12}+C_{11}+4 C_{44}}\right) \epsilon_{\|},
$$

where $d_{v}$ is the uniaxial deformation potential for the strain components with rhombohedral symmetry. For (111) samples, the strain tensor components $\epsilon_{i j}(i, j=x, y, z)$ with $i \neq j$ that enter the strain Hamiltonian ${ }^{23}$ can be calculated as described in Ref. 25, and can be expressed in terms of $\epsilon_{\|}$ $=\left(a_{\mathrm{film}}-a_{\mathrm{GaAs}}\right) / a_{\mathrm{GaAs}}$ by diagonalizing the strain tensor.

Similarly to the case of the (100) sample, we have used Eqs. (8)-(10) to calculate the energies of the $\mathrm{LH}$ and $\mathrm{HH}$ resonances as a function of the $\mathrm{N}$ and In compositions for $\mathrm{In}_{x} \mathrm{Ga}_{1-x} \mathrm{As}_{1-y} \mathrm{~N}_{y}$ grown on (111)-GaAs. Following the procedure described above for the (100) sample, we find that for $x=20.5 \%$ and $y=3.3 \%$ the calculated energies of the $\mathrm{HH}$ and LH states coincide with the values measured by PR and yield lattice spacings that are compatible with those obtained by HRXRD in our (111) sample $\left[(\Delta d / d)_{\perp}=0.01206\right]$. As for the (100) sample, this result allows us to infer the composition of the film.

From the LH-HH splitting measured by PR in the (111) sample $(42 \mathrm{meV})$, we find that $d_{v}=-3.0$, which is sizably lower than the linearly interpolated value for (In, Ga)(As, N) for $x=20.5 \%$ and $y=3.3 \%\left(d_{v}=-4.55\right.$; see Table II). This result confirms the abnormal composition dependence of the shear deformation potentials in $(\mathrm{In}, \mathrm{Ga})(\mathrm{As}, \mathrm{N})$.

\section{C. $($ In, Ga) $(\mathrm{As}, \mathrm{N})$ grown on $(N 11) A-G a A s(N=3,4,5)$}

For samples grown on (N11) substrates $(N=3,4,5)$, the analysis of the PR data is considerably more complicated than for samples grown on (100) and (111) substrates, since the strain Hamiltonian is not diagonal in a representation where the $\langle N 11\rangle$ direction is taken as the $z$ axis (see Ref. 23 for the similar case of stress applied along the $\langle 110\rangle$ direction). The eigenenergies of the strain Hamiltonian can still be obtained by diagonalizing the strain Hamiltonian, although the resulting strain-split states will consist of mixtures of the $\mathrm{LH}$ and $\mathrm{HH}$ states for zero strain. For samples grown along directions with lower symmetry such as (N11), the diagonalization of the strain tensor may be done numerically by taking the strain components for pseudomorphic films grown on non-(100) substrates, the calculation of which is described in Ref. 25.

For the sake of simplicity, we take an alternative approach and calculate the energies of $\mathrm{LH}$ and $\mathrm{HH}$ states for the (N11) samples by realizing that, similarly to the case of stress applied parallel to (110) in zinc blende materials, ${ }^{23}$ the strain-induced shear component change of the eigenenergies can be expressed, up to first order, as a function of the corresponding shifts for the $\langle 100\rangle$ and $\langle 111\rangle$ directions,

$$
\delta E_{N 11} \simeq p \delta E_{100}+(1-p) \delta E_{111},
$$

where $p$ is a constant that can be derived by diagonalizing the strain Hamiltonian and expressing the resulting eigenenergies, up to first order, linearly in $\delta E_{100}$ and $\delta E_{100}$. Here, for simplicity [and similarly to the case of the elastic parameter of the films for the (N11) samples, see Eq. (4)], we approximate $p$ by linearly interpolating $\delta E_{N 11}$ between $\delta E_{100}$ and $\delta E_{111}$, 


$$
\delta E_{N 11} \simeq \delta E_{100}+\frac{\alpha}{54.74^{\circ}}\left(\delta E_{111}-\delta E_{100}\right) .
$$

For both GaAs and InAs, given the values of the elastic constants and the shear deformation potentials of these two compounds, ${ }^{18} \delta E_{100} \sim \delta E_{111}$, which indicates that the error introduced by assuming Eq. (14) is low in the case of dilute $(\mathrm{In}, \mathrm{Ga})(\mathrm{As}, \mathrm{N})$. Then, the eigenenergies for $(\mathrm{In}, \mathrm{Ga})(\mathrm{As}, \mathrm{N})$ grown on (N11)-GaAs can be evaluated by replacing $\delta E_{100}$ in Eqs. (9) and (10) by $\delta E_{N 11}$ as expressed in Eq. (14).

To calculate $E_{\mathrm{LH}}$ and $E_{\mathrm{HH}}$ in the (N11) samples, we use the shear deformation potentials $b_{v}$ and $d_{v}$ determined previously for the (100) and (111) epilayers, since the composition of all our samples is expected to be similar and, in any case, it is not possible to determine them independently from the HH-LH splittings measured by PR in the (N11) samples. As a consequence, the calculated and the experimental HH-LH splittings and lattice spacings for these samples need not coincide, in contrast to the (100) and (111) films, for which $b_{v}$ and $d_{v}$ were directly obtained from $E_{\mathrm{LH}^{-}} E_{\mathrm{HH}}$ and the HRXRD data. Thus, any difference between calculations and experimental results in the $(N 11)$ films will provide a measure of the validity of Eq. (14) and of the $b_{v}$ and $d_{v}$ values obtained for the (100) and the (111) samples. Then, we obtain the $\mathrm{N}$ and In compositions, of the (N11) samples as in the case of (100) and (111) samples, i.e., comparing calculated and experimental energies of $\mathrm{HH}$ and $\mathrm{LH}$ resonances with the condition that the corresponding lattice parameters be compatible with the HRXRD data.

Following this procedure, we find that for the (511) sample the calculated energies of both the HH and LH resonances are in very good agreement with the values determined by PR when $x \approx 20 \%$ and $y \approx 2.3 \%$. For this composition, we obtain that $(\Delta d / d)_{\perp}=0.0172$, in fairly good agreement with the HRXRD result $\left[(\Delta d / d)_{\perp, \mathrm{HRXRD}}\right.$ $=0.01736)$.

For the (411) film, we find that the calculated and the experimental values of the $\mathrm{LH}$ and $\mathrm{HH}$ resonances are best fitted when $x \approx 21.5 \%$ and $y \approx 3 \%$. In this case, the calculated LH-HH splitting is slightly higher (around $10 \mathrm{meV}$ ) than the value obtained from the PR spectra. For this sample, we obtain $(\Delta d / d)_{\perp}=0.0167$, slightly below the result obtained by HRXRD $\left.\left[(\Delta d / d)_{\perp, \mathrm{HRXRD}}=0.01735\right)\right]$.

For the (311) sample, we obtain a very good agreement between the calculated and the experimental values of $E_{\mathrm{LH}}$ and $E_{\mathrm{LH}}$ when $x \approx 20 \%$ and $y \approx 2.8 \%$. For this pair of $x$ and $y$ values, we find that $(\Delta d / d)_{\perp}=0.0150$, in very good agreement with the HRXRD result $\left[(\Delta d / d)_{\perp, \mathrm{HRXRD}}=0.01543\right]$.

These results show that the analysis of the PR data based on Eq. (14) provides good agreement between the calculated energies of the PR resonances, the corresponding lattice spacings, and the experimental PR and HRXRD data for all three (N11) samples. Only for the sample grown on the (411)-GaAs substrate do we find some discrepancy between the calculated and the experimental energies and lattice spacings. We estimate that this discrepancy yields an uncertainty of about $\pm 2 \%$ in the resulting In fraction, but it does not affect much the $\mathrm{N}$ concentration thus obtained since, as will be discussed in the next section, the band-gap energy of (In,
Ga)(As, N) is very sensitive to the $\mathrm{N}$ content. The poorer results that we find for the (411) sample may be a consequence of the approximations involved in Eq. (14) and/or of some unnoticed experimental error in the PR or the HRXRD measurements.

It should be noted that the values of In concentration that we have obtained for these three samples are congruent with the values that one would expect from the nominal composition of the films. This, together with the good agreement of calculated and experimental PR resonances and lattice spacings, indicates that our analysis provides a reliable means to assess the composition of the (N11) strained (In, Ga)(As, N) epilayers. Also, our results suggest that the $b_{v}$ and $d_{v}$ values obtained previously for the (100) and (111) samples are not artificial but reproducible, thus confirming the abnormal valence-band splitting of strained (In, Ga)(As, N).

\section{DISCUSSION}

We have seen that the combined analysis of PR and HRXRD measurements of strained (In, Ga)(As, N) thin films allows one to assess their composition even in samples grown along low-symmetry directions. Obviously, the determination of the In and $\mathrm{N}$ content of the layers has different sources of error. These are mainly originated by the fitting procedure to determine the energy of the PR resonances and also by the error of the parameters used in the calculations. It is noteworthy, however, that the energy of the $\mathrm{HH}$ and $\mathrm{LH}$ resonances is very sensitive to the $\mathrm{N}$ content of the samples, which is due to the strong dependence of the band gap of (In, Ga)(As, N) on N content. This can be appreciated in Fig. 4, where it can be seen that an absolute variation of $0.5 \%$ in the $\mathrm{N}$ content leads to variations in the energy of the PR resonances as high as $\sim 40 \mathrm{meV}$.

It should be noted that if we had investigated relaxed (In, $\mathrm{Ga})(\mathrm{As}, \mathrm{N})$ epilayers, the $\mathrm{LH}$ and $\mathrm{HH}$ states would have been degenerate. In that case, we would have determined the $\mathrm{N}$ and In concentration of the films from the energy of the $E_{-}$ subband, as measured by PR, using the BAC model in combination with the (relaxed) lattice spacings determined by HRXRD. In our strained films, we have been able to determine the valence-band deformation potentials as well, and we have observed the abnormal behavior of the valence band in strained dilute nitrides, as reported in previous works. ${ }^{14,15}$

Our PR data and subsequent analysis suggest that the In and $\mathrm{N}$ content of fully strained (In, Ga)(As, N) do not depend strongly on substrate orientation (see Table I). The In fraction that we find for all samples turns out to be close to the nominal values and, as a consequence, we conclude that the HRXRD data alone could have been used to obtain a first approximation of the composition of the films. However, when looked in more detail, our results reveal some variations of the In composition from sample to sample. In the particular case of the (100) film, we obtain $x \sim 22 \%$. This means that for this particular sample, the HRXRD method alone would have led to a much lower $\mathrm{N}$ content.

Regarding the incorporation of $\mathrm{N}$, we find that the $\mathrm{N}$ content of the samples grown on (511)A and (311)A surfaces is lower than that of the (100) and (111)A samples, which 
explains the higher energy of the PR resonances observed in the former [the (411)A sample, for which our analysis seems to yield poorer results, behaves somewhat different, with intermediate $\mathrm{N}$ content, a higher In content, and $E_{\mathrm{LH}}$ and $E_{\mathrm{HH}}$ values similar to those of the (100) and (111)A samples]. However, the variations of $\mathrm{N}$ content that we observe in our samples are not as strong as those previously observed in $(\mathrm{Ga}, \mathrm{As}) \mathrm{N}$ quantum wells grown at higher temperatures on (100)- and (111)B-GaAs. ${ }^{3}$ In that work, a much higher $\mathrm{N}$ incorporation was found in (111)A samples with respect to (100) samples, contrary to the present work, where we observe the highest $\mathrm{N}$ content in the (100) film. While In probably has a bearing on the surface reconstruction for the different growth directions, the incorporation of $\mathrm{N}$ may be higher in our samples due to the lower growth temperatures. Also, it must be remembered that in (111)A surfaces there is one bond available for $\mathrm{N}$ atoms, while there are two bonds available in (100) surfaces. ${ }^{3}$ Thus, taking into account only the number of bonds available, neglecting the distortion on the lattice introduced by $\mathrm{N}$ and the effect of In on the growth, one would expect a higher $\mathrm{N}$ incorporation in (100) samples than in (111)A samples, as we observe here. For the (N11) films, $\mathrm{N}$ incorporation is expected to be lower due to the lower symmetry of these growth directions.

On the other hand, we observe that the In fraction tends to be higher in the samples with higher $\mathrm{N}$ incorporation (see Table I), which could indicate that the incorporation of In is enhanced by the presence of $\mathrm{N}$. This is supported by the fact that, as already mentioned, the In content of our (100) sample, which has the highest $\mathrm{N}$ content, is fairly higher than the nominal value expected from the MBE calibration. Since it is not known whether the incorporation of In in (In, Ga)As depends on the growth direction, and bearing in mind the uncertainties of our analysis, we conclude that the present data does not allow one to ascertain if the incorporation of $\mathrm{N}$ in $(\mathrm{In}, \mathrm{Ga})(\mathrm{As}, \mathrm{N})$ only depends on the growth direction or if the incorporation of both $\mathrm{N}$ and $\mathrm{In}$ is affected by each other. Further investigations dealing with $(\mathrm{Ga}, \mathrm{As}) \mathrm{N}$, (In, Ga)As, and $(\mathrm{In}, \mathrm{Ga})(\mathrm{As}, \mathrm{N})$ thin films grown on different non-(100) substrates with different $\mathrm{In}$ and $\mathrm{N}$ concentrations are required to understand the role of the growth direction on the incorporation of $\mathrm{N}$ and $\mathrm{In}$ into these materials.

Finally, we would like to stress that we have assumed Vegard's law to calculate the lattice parameter of (In, Ga)(As, N), $a_{\text {InGaAsN }}$, and analyze our PR and HRXRD data. As recently shown, the lattice parameter of $\mathrm{Ga}(\mathrm{As}, \mathrm{N})$ deviates slightly from Vegard's law for $y \geq 3 \%{ }^{8,9}$ Thus, it may be expected that $a_{\mathrm{InGaAsN}}$ is also higher than predicted by Vegard's law in our samples. However, such differences cannot be readily included in the calculations because there is no data available on the lattice parameter of $\operatorname{In}(\mathrm{As}, \mathrm{N})$. Taking into account the lattice parameters reported for $\mathrm{Ga}(\mathrm{As}, \mathrm{N})$ in Ref. 8 and assuming Vegard's law to calculate the lattice parameter of $\operatorname{In}(\mathrm{As}, \mathrm{N})$, we estimate that our analysis could be slightly underestimating $y$ (around an absolute $0.1 \%$ ) and overestimating $x$ (around an absolute 1\%). These errors, which obviously would have also been introduced if only the HRXRD data had been used to determine $x$ and $y$, are systematic from sample to sample. As a consequence, the rela- tive differences between the $\mathrm{N}$ and In compositions that we have obtained in our samples would have been preserved if a more accurate model for $a_{\mathrm{InGaAsN}}$ had been used. Thus, we conclude that our analysis is basically not affected by a lack of knowledge of the lattice parameter of (In, Ga)(As, N). However, it must be noted that with a higher lattice parameter, the resulting In concentration in the (100) sample would have been closer to the nominal value $(20 \%)$. This fact, if confirmed, would support the idea that the HRXRD data alone, provided that appropriate lattice parameters are considered, may be used to assess the composition of (In, $\mathrm{Ga})(\mathrm{As}, \mathrm{N})$ regardless of the substrate orientation, which is relevant for the day-to-day characterization of structures based on this material.

\section{CONCLUSIONS}

We have used a combination of PR and HRXRD measurements to determine the $\mathrm{N}$ and In compositions of $\mathrm{In}_{x} \mathrm{Ga}_{1-x} \mathrm{As}_{1-y} \mathrm{~N}_{y}$ thin films $(x \sim 20 \%, \quad y \sim 3 \%)$ grown pseudomorphically on (100) and non-(100) GaAs. We have shown that a combined analysis of the PR and HRXRD data allows one to evaluate the composition of the films. Such analysis may be applied to any quarternary alloy grown on (100) or non-(100) substrates, for which the determination of the composition by means of HRXRD alone is in general not possible.

Our data and analysis suggest that the incorporation of In in $(\mathrm{In}, \mathrm{Ga})(\mathrm{As}, \mathrm{N})$ depends very little on substrate orientation and does not differ much from that of (In, Ga)As. On the other hand, we find that the incorporation of $\mathrm{N}$ in (In, $\mathrm{Ga})(\mathrm{As}, \mathrm{N})$ exhibits some dependence on the growth direction. In particular, we find that the $\mathrm{N}$ content of $(N 11)$ samples $(N=3,4,5)$ is lower than for the (100) and (111) samples. However, the observed differences are much weaker than those previously observed in $\mathrm{Ga}(\mathrm{As}, \mathrm{N})$. Our analysis allows us to determine shear deformation potentials for our samples, which turn out to be sizably different from those obtained by linearly interpolating from the values of the pure binary compounds. This effect, already observed in $\mathrm{Ga}(\mathrm{As}, \mathrm{N})$, implies that the valence band of dilute nitrides is also perturbed by $\mathrm{N}$.

\section{ACKNOWLEDGMENTS}

This work supported by the Spanish Ministry of Education and Science (Project No. MAT 2004-0664 and Ramón y Cajal Program) and by the Engineering and Physical Sciences Research Council, United Kingdom. This work was supported in Poland by the Foundation for Polish Science through a Subsidy 8/2005.

\footnotetext{
${ }^{1}$ Semicond. Sci. Technol. 17(8) (2002), special issue on dilute nitrides; J. Phys.: Condens. Matter 16(31) (2004), special issue on dilute nitrides.

${ }^{2}$ S. Blanc et al., IEE Proc.: Optoelectron. 150, 64 (2003).

${ }^{3}$ A. Arnoult, F. Gonzalez-Posada, S. Blanc, V. Bardinal, and C. Fontaine, Physica E (Amsterdam) 23, 352 (2004).

${ }^{4}$ J. M. Sanchez, A. Guzman, J. M. Ulloa, A. Hierro, and E. Muñoz, Appl. Phys. Lett. 84, 2524 (2004).

${ }^{5}$ J. M. Sanchez et al., J. Cryst. Growth 270, 62 (2004).

${ }^{6}$ C. Mailhiot and D. L. Smith, Phys. Rev. B 35, 1242 (1987).

${ }^{7}$ W. Li, M. Pessa, and J. Likonen, Appl. Phys. Lett. 78, 2864 (2001).
} 
${ }^{8}$ W. J. Fan, S. F. Yoon, T. K. Ng, S. Z. Wang, W. K. Loke, R. Liu, and A. Wee, Appl. Phys. Lett. 80, 4136 (2002).

${ }^{9}$ W. J. Fan, S. F. Yoon, W. K. Cheah, W. K. Loke, T. K. Ng, S. Z. Wang, R. Liu, and A. Wee, J. Cryst. Growth 268, 470 (2004).

${ }^{10}$ J. F. Geisz, D. J. Friedman, J. M. Olson, S. R. Kurtz, and B. M. Keyes, J. Cryst. Growth 195, 401 (1998).

${ }^{11}$ A. Y. Polyakov et al., Solid-State Electron. 46, 2147 (2002).

${ }^{12}$ W. Shan, W. Walukiewicz, J. W. Ager III, E. E. Haller, J. F. Geisz, D. J. Friedman, J. M. Olson, and S. R. Kurtz, Phys. Rev. Lett. 82, 1221 (1999). ${ }^{13}$ W. Shan et al., Phys. Status Solidi B 223, 75 (2001).

${ }^{14}$ Y. Zhang, A. Mascarenhas, H. P. Xin, and C. W. Tu, Phys. Rev. B 61, 4433 (2000).

${ }^{15}$ M. H. Ya, Y. F. Chen, and Y. S. Huang, J. Appl. Phys. 92, 1446 (2002).

${ }^{16}$ J. Misiewicz, P. Sitarek, G. Sek, and R. Kudrawiec, Mater. Sci. 21, 263
(2003).

${ }^{17}$ J. Hornstra and W. J. Bartels, J. Cryst. Growth 44, 513 (1978).

${ }^{18}$ I. Vurgaftman, J. R. Meter, and L. R. Ram-Mohan, J. Appl. Phys. 89, 5815 (2001).

${ }^{19}$ I. Vurgaftman and J. R. Meter, J. Appl. Phys. 94, 3675 (2003).

${ }^{20}$ S. Adachi, J. Appl. Phys. 53, 8775 (1982).

${ }^{21}$ D. E. Aspnes, Surf. Sci. 37, 418 (1973).

${ }^{22}$ F. H. Pollak, in Modulation Spectroscopy of Semiconductors and Semiconductor Microstructures, Handbook on Semiconductors Vol. 2, edited by T. S. Moss (Elsevier Science, Amsterdam, 1994), pp. 527-635.

${ }^{23}$ F. H. Pollak and M. Cardona, Phys. Rev. 172, 816 (1968).

${ }^{24}$ H. Asai and K. Oe, J. Appl. Phys. 54, 2052 (1983).

${ }^{25}$ K. Yang, T. Anan, and L. J. Schowalter, Appl. Phys. Lett. 65, 2789 (1994). 